\title{
Analysis on the Safety of Expressway Interchange
}

\section{Jinxi Ding*}

China Merchants Chongqing Communications Technology Research \& Design Institute Co., Ltd., Chongqing 400067, China

\begin{abstract}
Currently, expressways encourage the use of interchange design methods in the layout mode to improve the safety of expressway operation in order to relieve road transportation pressure and strengthen the prevention of traffic safety accidents. The advantages and characteristics of the interchange method are more obvious and have important application value when compared to other interchange methods. This paper mainly studies and analyzes on the safety design and major challenges of interchanges in order to ensure the safe application of interchange forms in expressways.
\end{abstract}

Keywords: Expressway; Interchange; Safety

Publication date: May, 2021; Publication online: 31 May, 2021

*Corresponding author: Jinxi Ding, 270872203@qq.com

\section{Introduction}

Since the reform and opening-up, China's socioeconomic level has been significantly improved. Under such an evolution, people have put forward new requirements for the basic functions and comfortable experience on the road. In view of the current highway construction situation, the traditional highway interchange method has been difficult to adapt to the current development especially with the continuous improvement of highway construction level. In order to ensure the quality and safety of expressway operation and appropriate traffic flow, designers encourage the use of interchange design methods to optimize the layout and structure of expressways. During the specific design process, designers must combine safety performance design requirements, actively combine expressway network and interchange characteristics, scientifically position expressway operational functions, as well as establish a scientific and appropriate interoperable safety design plan during the specific design process.

2 Analysis of the Components and Major Challenges of Expressway Interchange Safety Design

In order to ensure that the quality of expressway operation can be enhanced and strengthened, designers should proactively base themselves on the safety performance design standards in the process of applying the interchange design mode, and conduct major research and analysis on the factors that affect the safety of the interchange from multiple aspects. Simultaneously, based to the analysis and feedback results, the relevant major components are planned and deployed in a reasonable manner to ensure that the safe operation of the expressway interchange can achieve the expected effect $^{[1]}$. Based on previous design experience, the safety design of expressway interchanges can be studied and analyzed from the following points.

\subsection{Entry and exit placements}

The placement of entrances and exits can be regarded as an important component of the expressway interchange's safety design system. In the specific design process, the designer should set a scientific and reasonable distance between the entrances and exits of adjacent main lines that communicate with each other as there are safety driving requirements for interchanges. In general, the distance adjustment needs to be scientifically set according to the length of the driving interlace 
and the length of the shifting lane. At the same time, it needs to be set according to the traffic sign layout requirements and the driver's response time. Based on previous design experience, if adjacent interchanges are too close to each other, it is difficult to ensure that the expected spacing requirements are met, hence designers can combine them and create an interchange form for application. For example, designers can place correspondingly separated collection and distribution lanes next to the through-travel lanes of a compound interchange.

By cascading the exits and entrances, the purpose is to ensure that only a pair of entrances and exits remain in the main line's driving direction, reducing the problem of excessive traffic and scattered traffic that existed previously. Designers can use measures such as three-dimensional separation between ramps to reduce the interweaving phenomena of highway mainstream ramps in the intercommunication compound interchange form with high traffic volume to ensure driving safety. In addition, if the main route has exits and entrances, it is essential that the designer creates a warning effect by determining and perfecting the signal signs to avoid dangerous problems during the vehicle's driving process. When necessary, the designer can use the method of merging multiple exits in the interchange design to reduce the problem of information confusion, as long as the safety design standards are met ${ }^{[2]}$.

\subsection{Variable speed lane design}

The variable speed lane can basically be regarded as an additional lane between the main line lane and the ramp. The main line's main function is to provide entrances and exits for vehicles entering and exiting the main line. Additional lanes for acceleration and deceleration are provided for the driving process by maintaining vehicle speed conditions that correspond to the ramp alignment. This section is prone to traffic accidents because the vehicles in this section need to change lanes or their vehicle speed.

To prevent traffic accidents at the position of the variable speed lane, it is recommended that the designer always adhere to the human-oriented and safety-oriented concept requirements, as well as scientifically calculate the shortest acceleration and deceleration length for vehicle driving safety under the premise of meeting the interchange's safety evaluation requirements. According to the calculation feedback results, measures such as placing deceleration belts in the deceleration lane are taken to encourage the vehicle to achieve the deceleration effect during the driving process ${ }^{[3]}$.

\subsection{Safety coordination design of ramp curve alignment index and vehicle operating speed}

From an objective point of view, whether the alignment indicators of interchanges and the operating speed of vehicles reach a coordinated state of operation will often have a vital impact on the safety and quality of expressway interchanges. Based on previous safety design experience, in order to ensure that the ramp curve alignment index and the vehicle operating speed is always in a safe and coordinated state, designers need to conduct appropriate research and analysis on the geometric elements of the curve at different locations according to the speed performance of different parts of the ramp. It should be noted that the speed used by the driver during the driving process is mainly adjusted according to the road conditions, or the safe speed is appropriately determined according to their own conditions. Therefore, in terms of specific design, designers should follow the human-oriented concept and fully consider the intellectual needs of the driver. In the selection of ramp curve alignment indicators, the actual operating speed should be used as the standard to design a scientific and appropriate ramp curve.

\subsection{Safety design analysis of ramp longitudinal slope}

In general, a problem with the longitudinal sight distance between the exit position and the entrance position, or an extremely wide ramp longitudinal slope, is likely to jeopardize expressway driving safety. For example, when the vertical surface of the expressway shows a curved and variable slope section with a small vertical curve radius, the driver's line of sight may be affected by certain 
obstructions and the problem of poor sight distance may occur. In view of this problem, the designer should focus on the safety design at the changing position of the longitudinal slope at the end of the ramp, whereby the designers can use a larger vertical curve radius to optimize the design effect ${ }^{[4]}$.

At the same time, when the main lane under the freeway ramp appears as a curved ramp section, the designer should appropriately increase the vertical curve radius for safety design considerations in order to provide drivers with good visual conditions. In addition, the design of the ramp longitudinal slope should be as smooth as possible to avoid unnecessary reverse slope problems. Thus, there should be room for the design of the largest longitudinal slope, whereas for the minimum longitudinal slope design the longitudinal drainage requirements should be considered.

\subsection{Superelevation safety design of ramp}

For the safety design of expressway interchanges, the superelevation safety design of ramps can basically be regarded as an important factor affecting the safety of expressway interchanges. In the specific design process, the designer should appropriately determine the ramp's superelevation value based on the variable driving speed on the ramp. At the same time, in the ramp superelevation design process, the designer should take the initiative to set the superelevation design components according to the environmental conditions and traffic flow conditions of the project location. It should be noted that if the ramp joins from the outside of the superelevation section of the main line, the designer should set the superelevation gradient in the deceleration lane section to a reasonable level.

\section{Other Suggestions}

In order to further improve the safety of expressway interchange design, it is recommended that designers should set a scientific and appropriate interchange lane type according to the highway environment and surrounding topographic features. At the same time, to reduce driver tiredness, a certain amount of greenery should be planted within a certain range of the road. The road traffic control facilities should be fully equipped to prevent accidents for the safety of the drivers during driving. It should be noted that traffic control facilities such as traffic signals must be correct and clear, and signs must be placed in a conspicuous position. For weather conditions such as the rainy season, construction personnel should promptly remove water from the road surface to avoid accidents for the safety of the drivers. In addition, in the design of interchange ramps, designers should rationally design the ramp radius and linearity according to design requirements, to fundamentally improve the design level of the expressway interchanges.

\section{Conclusion}

In conclusion, it is recommended that designers should proactively focus on the safety design concept, and make overall planning and appropriate deployment for the safety design of expressway interchanges from multiple aspects, in order to reduce safety accidents on expressway interchanges. Simultaneously, take the initiative to execute relevant important components from the perspective of safety design and management. Furthermore, to avoid expressway interchange safety design effects failing to fulfil expectations, it is advised that designers should combine advanced design knowledge from both locally and internationally to change and optimize current deficiencies in the expressway interchange safety design in a timely manner. As a result, it has the potential to significantly improve the safety of highway operations in our country.

\section{Disclosure statement}

The author declares no conflict of interest. 


\section{References}

[1] Zhou, T., Yu F., Zhang, X., Liu, M.Y(2019). The Location and Cause Analysis of Frequent Accidents of Expressway Horn-Shaped Interchange[J]. Highway Traffic Technology,35(01): 129-133+139.

[2] Yang, F.Y., Zhao, Y., Zhang, Y.F(2019). Analysis of Traffic Accident Characteristics of Expressway Interchanges[J]. Transportation Science and Technology,(02):107-110.
[3] Li, B.Q(2019). Research on the Main Points of the Safety Evaluation of Interchanges[J]. Building Materials and Decoration,(19):243-244.

[4] Sheng, X.F(2019). Analysis of the Main Points of the Safety Evaluation of Interchanges[J]. Green Environmental Protection Building Materials,(05): 100 